Urologe 2021 · 60:241-243

https://doi.org/10.1007/s00120-021-01467-y

Angenommen: 22. Januar 2021

Online publiziert: 8. Februar 2021

(c) Springer Medizin Verlag GmbH, ein Teil von Springer Nature 2021

Das moderne Pflegeverständnis hat sich über die letzten Jahrzehnte stark verändert. Dabei hat sich der Caring Begriff als ein zentraler Bestandteil in der Disziplin der Pflege entwickelt, denn die fürsorgende Aufgabe kann erst durch das in Beziehung treten mit Anderen entstehen. Besonders die Interaktion mit Menschen wird damit zur Voraussetzung für eine professionelle Pflege.

Die aktuelle Covid-19-Pandemie stellt Politik und Gesellschaft sowie die Gesundheitssysteme der Länder vor besondere Herausforderungen [14]. Viele Veränderungen in der Gesellschaft, bedingt durch ein hohes Maß an Ungewissheit und Ängsten, sind die Konsequenz. Auch im Pflegeberuf kommt es zu diversen Einschränkungen, die einen Einfluss auf den Arbeitsalltag aller Betroffenen nehmen.

„Professionell Pflegende stellen die größte Berufsgruppe des Gesundheitssystems dar und tragen maßgeblich zu der Versorgungssicherheit der Patienten und zur Bewältigung der Pandemie bei“ [14, S. 60]. Ebenso stehen Pflegepersonen, im Vergleich zu jeder anderen Gesundheitsprofession, die längste Zeit im direkten Patientenkontakt [7]. Somit sind sie in ihrer täglichen Arbeit mit einem besonders hohen Ansteckungsrisiko konfrontiert [3]. Das ständige Infektionsrisiko prägt den Arbeitsalltag von Pflegepersonen in Zeiten der Covid19-Pandemie ebenso wie eine erhöhte

Dieser Beitrag wurde zuerst publiziert in Gabl, K., Küpper, A. \& Pöhner, J. Nähe auf Distanz. Pflegez 73, 58-60 (2020). https://doi.org/10. 1007/s41906-020-0920-7.

Katharina Gabl · Alexander Küpper · Julien Pöhner

Universität Wien, Wien, Österreich

\title{
Nähe auf Distanz
}

\section{Pflege als Caring-Disziplin in Zeiten der Covid-19-Pandemie}

Arbeitslast, begrenzte materielle und personelle Ressourcen, soziale Isolation und ein Bruch in den täglichen Routinen [15].

\section{Pflege: Dienst an der Menschheit}

Die Pflege ist, wie andere Berufe des Gesundheitswesens auch, ein Dienst an der Menschheit [26]. „Pflegekunde beruht auf menschlicher Sorge, die ihre spezifische Gestalt durch die berufliche Aufgabe erhält" [23, S. 15]. Pflege ist eine Caring Disziplin und somit eine fürsorgende Aufgabe, die sich erst durch in Beziehung treten mit Anderen etabliert [17].

Die ersten Gedanken des Carings als Grundlage professioneller Pflege wurden Anfang der 1980iger Jahre in Theorien des angloamerikanischen Raumes aufgenommen und entwickelt [24]. In der Folge wurde ein erstes Modell im Rahmen des Caring Prozesses von Swanson (1991) veröffentlicht. Die Ergebnisse einer Meta-Synthesis von Fingfeld-Connett [10] zeigen, dass das Konzept Caring ein kontextbezogener und persönlicher Prozess ist, der durch das pflegerische Tun, eine zwischenmenschliche Sensitivität, sowie intime Beziehungen charakterisiert ist. Die zwischenmenschliche Sensitivität als Grundlage für den Caring Prozess erfordert die physische und emotionale Präsenz der Pflegenden. Aktives Zuhören, Blickkontakt, Berührungen und Gespräche können den Grundstein für die intime Beziehung bilden, die zwischen der Pflegeperson und den zu Pflegenden entstehen soll. Durch den Caring Prozess wird das physische und psychische Wohlbefinden der Patienten gesteigert.
Auch die mentale Gesundheit der Pflegepersonen kann vom Caring Konzept profitieren [10].

Der Caring Begriff ist ein zentraler Bestandteil des modernen Pflegeverständnisses, welches sich über Jahrzehnte entwickelt hat und definiert sich über die Handlung aber auch durch die Haltung des Pflegenden [11, 12, 17]. Auch Wojnar [26] beschreibt Caring als die wertschätzende Bezogen- und Verbundenheit, sowie die Verantwortung anderen unterstützungsbedürftigen Menschen gegenüber. „The nurse-patient relationship is the essence of caring " [17, S. 90]. Die Interaktion mit Menschen wird damit zur Voraussetzung für professionelle Pflege [25].

\section{Kommunikation als Form der sozialen Interaktion}

„Der Begriff der Interaktion stellt einen Sammelbegriff für all jene Prozesse dar, die zwischen zwei oder mehreren Personen in einem sozialen Kontext ablaufen" [8, S. 24]. Die Kommunikation als Form der menschlichen Interaktion setzt sich sowohl aus verbalen Äußerungen als auch aus nonverbalen Signalen zusammen (ebd.). Diese umfassen Mimik, Blickverhalten, Gestik und Bewegung, Haltung, Berührung, das Verhalten im Raum, das individuelle Erscheinungsbild, lautliche Äußerungen und auch den Geruch [1]. Aber auch der Umgang mit Gefühlen ist ein wichtiger Aspekt in der zwischenmenschlichen Interaktion. Bewusste und unbewusste Emotionen können den Kommunikationsprozess maßgeblich beeinflussen. Stress, Ängste und Ungewissheit wirken sich negativ auf den Beziehungsaufbau aus [22]. Somit 
hat „alles Verhalten in interpersonalen Situationen Mitteilungscharakter [...]“ (Graumann, 1972; zit. aus [8, S. 22]).

\section{Veränderungen im Pflegealltag}

Seit dem Ausbruch der Coronakrise hat sich die Berufswelt in der Pflege drastisch verändert. Fast täglich werden vom Robert Koch-Institut (RKI) in Deutschland, der Medizin und Virologie aus der ganzen Welt sowie den Hygieneabteilungen der Krankenhäuser neue Vorschriften zum Umgang mit den Betroffenen herausgegeben. Folglich ist das Betreten des Zimmers auf das notwendige Maß für die pflegerische und medizinische Versorgung zu reduzieren. Die Unterbringung der Betroffenen sollte nach Möglichkeit nur in Einzelzimmern stattfinden. Das Betreten des Zimmers ist ausschließlich mit kompletter Schutzausrüstung (Haube, Maske, Brille, Kittel, Handschuhe, ggf. Face Shield) gestattet und die Pflegehandlungen sollen zusammengefasst werden [16, 18, 21].

Diese im Arbeitsalltag geforderten Einschränkungen stellen Pflegepersonen vor verschiedenste neue Herausforderungen. Durch die notwendigen Isolationsmaßnahmen ist die Durchführung von pflegerischen Tätigkeiten zunehmend erschwert. Die Bedürfnisse der zu pflegenden Personen nach Austausch, Zuwendung und Nähe kann nur noch in geringem Maße gestillt werden. Ebenso wie die Pflege des Betroffenen, bezieht Pflege als Profession auch die Zusammenarbeit mit den Angehörigen ein. Doch die Betreuung ist erschwert und gestaltet sich in der aktuellen Zeit durch allgemeine Besuchsverbote deutlich eingeschränkt [19].

Auch in Zeiten der Covid-19-Krise wird das Streben der Pflegepersonen nach Interaktionen, trotz einer damit einhergehenden Gefährdung der eigenen Gesundheit, nicht gemindert [25]. Ein weiterer Stressfaktor im Arbeitsalltag wird durch den weltweiten Mangel an Schutzausrüstung und der somit steigenden Angst vor einer Selbstinfektion verursacht [19].

\section{Interaktion als Grundlage professioneller Pflege}

Interaktionen, die einen wesentlichen Bestandteil des Caring Prozesses bilden, werden durch die begrenzten zeitlichen Ressourcen der Pflegepersonen beeinflusst. Gespräche werden auf ein Minimum, im Rahmen der pflegerischen Tätigkeiten, begrenzt und somit ist ein adäquater Beziehungsaufbau zwischen Patienten und Patientinnen und der Pflege nur bedingt möglich. Die Kommunikation zwischen den Interagierenden wird aber nicht nur durch den Faktor Zeit, sondern ebenso durch Isolationsund Schutzmaßnahmen eingeschränkt. Durch das Tragen von Schutzkleidung werden das Gesicht und der Körper der Pflegeperson größtenteils verborgen. Hierdurch können der Gesichtsausdruck und auch die Lippenbewegungen des Gegenübers nur vermutet und Körperbewegungen nur schwer erkannt werden. Ebenso wird das individuelle Erscheinungsbild der Pflegepersonen und ihre Haltung im Raum durch die Schutzkleidung fast komplett verdeckt und persönliche Gerüche werden durch die Krankenhausumgebung beeinflusst. Berührungen werden weitestgehend vermieden. Wenn sich die Interagierenden jedoch berühren, so geschieht dies nur mit Handschuhen. Durch diese bestehenden Schutzmaßnahmen werden Gefühle der Fremdheit, Bedrohung und Distanz vermittelt. Als Resultat werden somit zwischenmenschliche Kommunikationsmuster erheblich eingeschränkt und ein vertrauter Beziehungsaufbau zwischen Betroffenen und Pflegekräften zunehmend erschwert [13].

\section{Diskussion - Was bietet die Forschung?}

Auf Grund der Aktualität der Pandemie existiert nur sehr wenig evidenzbasierte wissenschaftliche Literatur zu der Thematik. Laut Helmbold und Schäfer [14] bewegen sich die meisten der bisher publizierten Arbeiten in den Bereichen der Medizin und der Virologie. Aus der bestehenden Literatur lässt sich ableiten, dass Handlungsabläufe in der Pflege umgestellt und angepasst, ganze Stationen umgebaut und neu aufgerüstet werden müssen. In vielen Ländern Europas übernehmen Pflegepersonen Aufgaben, über die sie nicht ausreichend Kenntnis besitzen, wodurch die Arbeitsbelastung in ein unermessliches Maß steigt. Durch die zusätzliche Reduzierung des direkten $\mathrm{Pa}$ tientenkontaktes zum Schutz des Personals und zur Ressourceneinsparung, sind die Erkrankten in vielen Situationen auf sich alleine gestellt $[2,5,9,16,20,21]$.

Vordergründig gilt es, in der aktuellen Zeit das Ansteckungs- und Übertragungsrisiko des Covid-19-Virus durch entsprechende Handlungen und Maßnahmen zu minimieren. Dennoch ist es weiterhin ein primäres Ziel, neben der kompetenten Versorgung der Patienten und Patientinnen, weitgehend auch die Normalität im Pflegeprozess sowie auf der Beziehungsebene aufrechtzuerhalten, so dass professionelle Pflege auch auf der Caring Ebene stattfinden kann. Doch durch die im Arbeitsalltag geforderten Einschränkungen wird die professionelle Pflege vor viele Herausforderungen gestellt.

Neben den notwendigen Isolationsmaßnahmen, der mangelnden Zeit des Personals und der Reduzierung der pflegerischen Tätigkeiten auf ein Minimum, wird durch die Notwendigkeit von Schutzkleidung die Interaktion mit den Patienten und Patientinnen deutlich erschwert. Des Weiteren sind die Arbeit und das Verhalten der Pflegepersonen durch psychische Faktoren stark beeinflusst. Stress stellt eine zusätzliche Barriere in der Interaktion und somit im Caring Prozess dar. Auch Angst und Ungewissheit sind tägliche Begleiter im Arbeitsleben, die durch Gegebenheiten wie dem weltweiten Mangel an Schutzkleidung deutlich gefördert werden [4].

\section{Die Pflegearbeit als fürsorgende Caring Aufgabe und die Eindämmung des Virus stehen im Konflikt}

Der eigentlich selbstverständliche und gewohnte Umgang mit den Betroffenen sowie die Handlungen innerhalb der Caring Disziplin Pflege, sind durch die Covid-19-Maßnahmen und damit einhergehenden Faktoren zusehends 
eingeschränkt. Die professionelle Pflege erfährt durch die globale Pandemie erhebliche Einschnitte in ihrer Selbstverständlichkeit und Professionalität. Somit stehen die Pflegearbeit als fürsorgende Caring Aufgabe und die Eindämmung des Virus im Konflikt. Der schmale Grad zwischen so viel Interaktion, dass professionelle Pflege stattfinden kann und so wenig, dass das Ansteckungsrisiko weitestgehend reduziert wird, stellt eine wesentliche Herausforderung des Arbeitsalltages von Pflegenden in Zeiten der Coronakrise dar [6]. Befriedigende Lösungen für eine Nähe auf Distanz sind gefordert und für eine künftige komplementäre Versorgung im stationären wie auch im ambulanten Sektor und Langzeitpflegebereich unumgänglich.

\section{Fazit}

Um eine Pandemie bekämpfen zu können, bedarf es einer Vielzahl an Maßnahmen. Diese müssten im Vorhinein geplant werden, so dass das Gesundheitssystem in der Krisensituation bestmöglich bestehen kann.

Alle Maßnahmen können jedoch die zwischenmenschliche Interaktion und das in Beziehung treten mit Anderen nicht ersetzen. Eine weltweit auftretende Erkrankung gefährdet immer einen wesentlichen Bestandteil der Profession Pflege - hier die Pflege als Caring Disziplin.

Die Coronakrise sollte als Chance gesehen werden, die Herausforderungen zu erkennen und entsprechende Schulungsund Interventionsleitlinien für den Umgang mit Schutzmaßnahmen oder Ressourcenknappheit, aber auch Faktoren wie Stress, Angst und Ungewissheit, zu erarbeiten.

\section{Korrespondenzadresse}

\section{Alexander Küpper, BSc}

Universität Wien

Wien, Österreich

cpakuepper@gmx.de

\section{Einhaltung ethischer Richtlinien}

Interessenkonflikt. K. Gabl, A. Küpper und J. Pöhner geben an, dass kein Interessenkonflikt besteht.

Für diesen Beitrag wurden von den Autoren keine Studien an Menschen oder Tieren durchgeführt. Für die aufgeführten Studien gelten die jeweils dort angegebenen ethischen Richtlinien.

\section{Literatur}

1. Argyle M (2013) Körpersprache \& Kommunikation. Nonverbaler Ausdruck und Soziale Interaktion, 10. Aufl. Junfermann, Paderborn

2. Begerow A, Gaidys U (2020) COVID-19 Pflege Studie. Erfahrungen von Pflegenden während der Pandemie - erste Teilergebnisse. In: Pflegewissenschaft, Sonderausgabe: Die Corona-Pandemie, S 33-36

3. Bergeron SM, Cameron S, Armstrong-Stassen M, Pare K (2006) Diverse implications of a national health crisis: a qualitative exploration of community nurses' SARS experiences. Can J Nurs Res 38(2):42-54

4. Bohlken J, Schömig F, Lemke MR, Pumberger M, Riedel-Heller SG (2020) COVID-19-Pandemie: Belastungen des medizinischen Personals. Psychiat Prax 47(04):190-197

5. Bouadma L, Lescure FX, Lucet JC, Yazdanpanah Y Timsit JF (2020) Severe SARS-CoV-2 infections: practical considerations and management strategy for intensivists. IntensiveCare Med 46:579-582

6. Bournes DA, Ferguson-Paré M (2005) Preserving through a difficult time during the SARS Outbreak in Toronto. Nurs Sci Q 18(4):324-333

7. Chan S (2003) Nurses fighting against severe acute respiratory syndrome (SARS) in Hong Kong. J Nurs Scholarsh 35(3):209

8. Christmann U (1999) Kommunikation, Interaktion. Kölner Psychol Stud 4(1):22-26

9. Eufinger A (2020) Die COVID-19-Pandemie: Arbeitsrecht im Krankenhaus in bewegten Zeiten. MedR 38:450-457

10. Finfgeld-Connett D (2008) Meta-synthesis of caring in nursing. JClin Nurs 17(2):196-204

11. Fosbinder $D$ (1994) Patient perceptions of nursing care: an emerging theory of interpersonal competence. J Adv Nurs 20(6):1085-1093

12. Gillespie H, Kelly M, Duggan S, Dornan T (2017) How do patients experience caring? Scoping review. PatientEduc Couns 100(9):1622-1633

13. Halek M, Reuther S, Schmidt J (2020) Herausforderungen für die pflegerische Versorgung in der stationären Altenhilfe: Corona-Pandemie 2020. MMW Fortschr Med 162(9):51-54

14. Helmbold A, Schäfer A (2020) Covid-19 aus pflegediagnostischer Perspektive. In: Pflegewissenschaft, Sonderausgabe: Die Corona-Pandemie, S60-66

15. Jansen T (2020) Angst vor Corona - Bedrohung der Pflege durch die Logik eines Affekts. In: Pflegewissenschaft, Sonderausgabe: Die CoronaPandemie, S53-56

16. Liang T (2020) Handbook of COVID-19 prevention and treatment. The First Affiliated Hospital, Zhejiang University School of Medicine. Compiled according to clinical experience

17. Meleis Al (2018) Theoretical nursing. Development and process, 6. Aufl. Wolters Kluwer, Philadelphia

18. Pelz S, Kaltwasser A, Dubb R (2020) COVID19: Allgemeine Handlungsempfehlungen kurz zusammengefasst. In: Pflegewissenschaft, Sonderausgabe: Die Corona-Pandemie, S38-40

19. Riedel A, Heidenreich T (2020) Der Applaus für die (Für)Sorge fordert die Protektion der Selbst(für)sorge. In: Pflegewissenschaft, Sonderausgabe: Die Corona-Pandemie, S156-162

20. Rieg S, Busch HJ, Hans F, Grundmann H, Biever P, Bürkle H, Hammer T, Thimme R, Kern W (2020) COVID-19-Versorgung - Strategien der Taskforce Coronavirus und Erfahrungen von den ersten 115 Fällen am Universitätsklinikum Freiburg. Dtsch Med Wochenschr 145(10):657-664

21. Robert Koch-Institut (2020) Empfehlungen des RKI zu Hygienemaßnahmen im Rahmen der Behandlung und Pflege von Patienten mit einer Infektion durch SARS-CoV-2. https://www.rki.de/ DE/Content/InfAZ/N/Neuartiges_Coronavirus/ Hygiene.html.Zugegriffen: 20. Mai 2020

22. Rogall R, Joskus H, Adam G, Schleinitz G (2005) Professionelle Kommunikation in Pflege und Management - Ein praxisnaher Leitfaden. Schlütersche Verlagsgesellschaft, Hannover

23. Schnepp W (1996) Pflegekundige Sorge. Pflege 2(96):13-16

24. Stemmer R (2003) Zum Verhältnis von professioneller Pflege und professioneller Sorge. In: DV Pflegewissenschaft (Hrsg) Das Originäre der Pflege entdecken. Pflege beschreiben, erfassen, begrenzen. Mabuse, Frankfurt am Main, S43-62

25. Wirth LM, Hülsken-Geisler M (2020) Wenn nicht jetzt, wann dann? In: Pflegewissenschaft, Sonderausgabe: Die Corona-Pandemie, S118-120

26. Wojnar DM (2009) Caring. In: Peterson SJ, Bredow TS (Hrsg) Middle range theories: application to nursing research, 2. Aufl. Lippincott Williams \& Wilkins, Philadelphia, S 189-201 